\title{
Quantum trajectories in elastic atom-surface scattering: Threshold and selective adsorption resonances
}

\author{
A. S. Sanz ${ }^{\text {a) }}$ \\ Chemical Physics Theory Group, Department of Chemistry, University of Toronto, \\ Toronto M5S 3H6, Canada \\ S. Miret-Artés ${ }^{\text {b) }}$ \\ Instituto de Matemáticas y Física Fundamental, Consejo Superior de Investigaciones Científicas, \\ Serrano 123, 28006 Madrid, Spain
}

(Received 1 June 2004; accepted 12 October 2004; published online 13 December 2004)

\begin{abstract}
The elastic resonant scattering of $\mathrm{He}$ atoms off the $\mathrm{Cu}(117)$ surface is fully described with the formalism of quantum trajectories provided by Bohmian mechanics. Within this theory of quantum motion, the concept of trapping is widely studied and discussed. Classically, atoms undergo impulsive collisions with the surface, and then the trapped motion takes place covering at least two consecutive unit cells. However, from a Bohmian viewpoint, atom trajectories can smoothly adjust to the equipotential energy surface profile in a sort of sliding motion; thus the trapping process could eventually occur within one single unit cell. In particular, both threshold and selective adsorption resonances are explained by means of this quantum trapping considering different space and time scales. Furthermore, a mapping between each region of the (initial) incoming plane wave and the different parts of the diffraction and resonance patterns can be easily established, an important issue only provided by a quantum trajectory formalism. (C) 2005 American Institute of Physics.
\end{abstract}

[DOI: $10.1063 / 1.1828032]$

\section{INTRODUCTION}

Resonance phenomena are a very important issue in scattering theory. In particular, in elastic atom-surface scattering there are only two types of quantum resonances of totally different nature: threshold resonances (TRs) and selective adsorption resonances (SARs). TRs were first reported for rotational diffraction by Sibener and co-workers. ${ }^{1}$ On the other hand, the selective adsorption has been a quite elusive effect because the corresponding intensities are too weak to be observed for weakly corrugated surfaces. Due to the great progress in the design of intense monoenergetic atom beam sources ${ }^{2}$ and the availability of well characterized stepped surfaces, ${ }^{3}$ together with new focused resonanceenhanced mechanisms, ${ }^{4}$ a very rich variety of data on SARs is now at our disposal.

For any in-plane elastic scattering process, the energy and parallel momentum conservation rules can be globally expressed as a function of the incident wave vector $\mathbf{k}_{i}$ as

$$
\mathbf{k}_{\mathbf{G}, z}^{2}=\mathbf{k}_{i}^{2}-\left(\mathbf{K}_{i}+\mathbf{G}\right)^{2},
$$

where the square wave vector quantities are given in energy units, with $\hbar^{2} / 2 m=1$ ( $m$ being the mass of the incident particle). Here, $\mathbf{k}_{\mathbf{G}, z}$ is the perpendicular-to-the-surface component of the outgoing wave vector, $\mathbf{K}_{i}$ is the parallel-to-thesurface component of $\mathbf{k}_{i}$ (with $\theta_{i}$ being the incidence angle measured from the outward normal to the surface), and $\mathbf{G}$ is a reciprocal lattice vector. The incidence conditions leading

\footnotetext{
a) Author to whom correspondence should be addressed. Electronic mail: asanz@chem.utoronto.ca

b)Electronic mail: s.miret@imaff.cfmac.csic.es
}

to TRs and SARs are easily obtained from the kinematic relation (1):

(i) TR condition:

$$
\mathbf{k}_{\mathbf{N}, z}^{2}=\mathbf{k}_{i}^{2} \cos ^{2} \theta_{f}=0,
$$

where $\mathbf{N} \in \mathbf{G}$ is the reciprocal lattice vector associated to an emerging/evanescent diffraction beam when the Bragg or final scattering angle is $\theta_{f}= \pm \pi / 2$. Therefore, threshold angles $\theta_{\mathrm{TR}}^{\mathrm{N}}$ correspond to critical incidences, such that for $\theta_{i}>\theta_{\mathrm{TR}}^{\mathbf{N}}$ the Bragg $\mathbf{N}$-diffraction beam or channel becomes unobservable.

The TR or emerging/evanescent beam is a very general feature of diffractive systems, and should be observed whenever the scattered particle incidence conditions are such that a new diffraction beam just appears/disappears. Within this context the term resonance is somewhat misleading since we are not dealing with real quasibound states. Due to the unitary condition of the scattering $S$-matrix, this effect manifests in the intensity of all diffracted peaks, although it may be very weak. TRs are expected to provide information about the long range part of the interaction potential and to be easily detectable in experiments for highly corrugated surfaces. ${ }^{5,6}$

A classical picture of a TR is provided by chaotic scattering in the light of a new phenomenon: the skipping singularity. ${ }^{6,7}$ The onset of classical trapping or classical chaos takes place when a (outgoing) surface rainbow angle reaches $\pm \pi / 2$. Then, the scattered particle undergoes a free motion parallel to the surface before leaving it, although its perpendicular motion remains bounded due to the attractive term of the surface interaction potential. This behavior cor- 


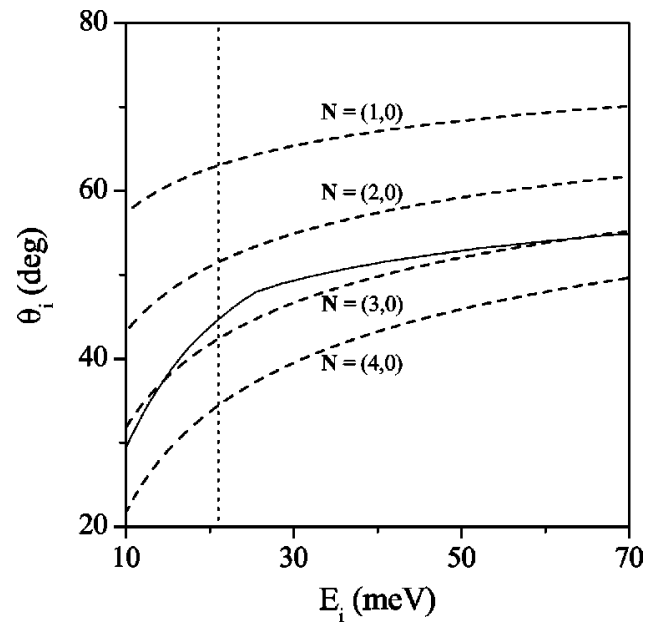

FIG. 1. TR conditions (dashed lines) for the reciprocal lattice vectors $\mathbf{N}$ $=(n, 0)$, with $n=1,2,3,4$ [in units of $2 \pi / a, a$ being the unit cell length of the assumed one-dimensional $\mathrm{Cu}(117)$ surface], and onset of chaos (solid line) as a function of the incidence energy and angle for the $\mathrm{He}-\mathrm{Cu}(117)$ system. The vertical dotted line (at $E_{i}=21 \mathrm{meV}$ ) indicates the incidence energy for which the results shown in this work were obtained.

responds to a new kind of singularity in the classical deflection function (the final deflection angle versus the impact parameter), that is, different from the one leading to the surface rainbow condition, which has been called the skipping singularity because it recalls the classical picture of "skipping" stones on the surface of a river. Hence, when the final classical scattering angle $\pm \pi / 2$ coincides with a Bragg or observable angle (condition for TR), this singularity also becomes observable and the corresponding diffraction pattern can be interpreted as a quantum manifestation of classical chaos. ${ }^{8}$

In Fig. 1 the onset of classical chaos (solid line) and the TR conditions (dashed lines) for the reciprocal lattice vectors $\mathbf{N}=(n, 0)$, with $n=1,2,3,4$ (in units of $2 \pi / a, a$ being the unit cell length), are plotted as a function of the incidence conditions for the scattering of He atoms from an assumed one-dimensional $\mathrm{Cu}(117)$ suface. At $E_{i}=21 \mathrm{meV}$ (vertical dotted line), above the onset of classical chaos, in the multiple scattering regime, the TR condition is fulfilled by the reciprocal lattice vectors $(1,0)$ and $(2,0)$; on the other hand, below the onset of classical chaos, in the single scattering regime, the TR condition is satisfied by $(3,0)$ and $(4,0)$. Here, the $(3,0)$ channel plays a key role in the dynamics of the system. Unlike any other channel, the dynamics associated to this one can be either chaotic or regular depending on the incidence conditions initially chosen. The intersection points between the onset of classical chaos and the $(3,0)$ channel satisfy simultaneously the TR requirement and the quantum rainbow condition, that is, $\pm \pi / 2$ is an observable Bragg angle. Therefore, for incidence conditions below the onset of chaos, the $(3,0)$ channel is classically forbidden, but energetically allowed, which means (from a semiclassical viewpoint) that only complex classical trajectories ${ }^{7}$ will contribute to the intensity of the corresponding diffraction beam. For incidence conditions above the onset of chaos, the $(3,0)$ channel is both classically and energetically allowed, and the contribution to its intensity is given by a set of homoclinic trajec-

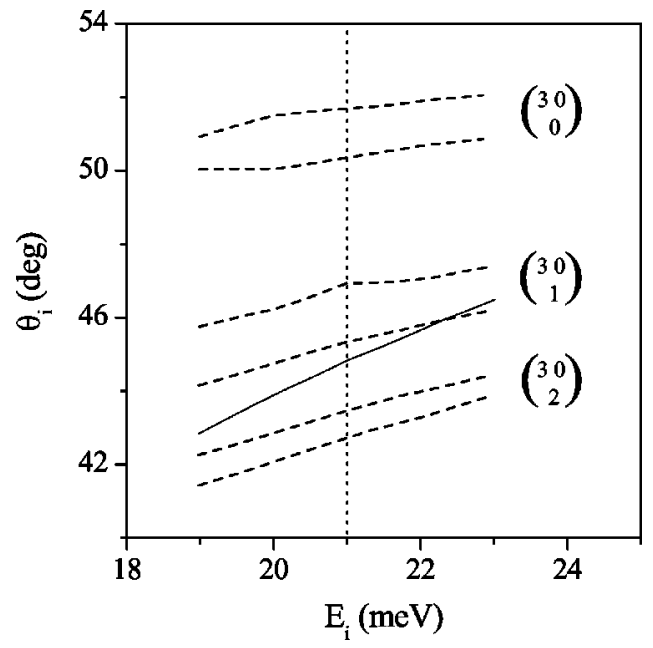

FIG. 2. SAR conditions (dashed lines) when the reciprocal lattice vector exchanged in the resonance process is $\mathbf{B}=(3,0)$ [in units of $2 \pi / a$, $a$ being the unit cell length of the assumed one-dimensional $\mathrm{Cu}(117)$ surface], and onset of chaos (solid line) as a function of the incidence energy and angle for the $\mathrm{He}-\mathrm{Cu}(117)$ system. Dashed lines represent here the positions plus/ minus the angular half-widths of the three bound states $\left(\epsilon_{n}\right.$, with $n$ $=0,1,2$ ) corresponding to each resonance. The vertical dotted line (at $E_{i}$ $=21 \mathrm{meV}$ ) indicates the incidence energy for which the results shown in this work were obtained.

tories that escape to the asymptotic region with $\theta_{f}=\pi / 2$ at $t=+\infty$. And finally, for initial conditions coinciding with the intersection points, the TR is associated to a quantum rainbow. $^{7}$

(ii) SAR condition:

$$
\mathbf{k}_{\mathbf{B}, z}^{2}=\mathbf{k}_{i}^{2}-\left(\mathbf{K}_{i}+\mathbf{B}\right)^{2}=-\left|\epsilon_{n}\right|,
$$

where $\mathbf{B} \in \mathbf{G}$ is the reciprocal lattice vector exchanged in the resonance process and $\epsilon_{n}$ is the $n$th bound state of the attractive, surface-averaged potential resulting along the perpendicular direction. Notice that now $\mathbf{B}$ corresponds to a closed or energetically forbidden diffraction channel. In Fig. 2 the boundary between the chaotic and regular regimes (solid line) as well as the three SARs for the $\mathbf{B}=(3,0)$ reciprocal lattice vector (in units of $2 \pi / a$ ) have been represented as a function of the incidence conditions in energy and angle for the $\mathrm{He}-\mathrm{Cu}(117)$ system. The SARs or incoming-particle quasibound states are caused by the presence of an attractive term in the interaction potential, and then are expected to provide information about the well region of this potential. In contrast to TRs, SARs manifest as anomalous intensities in all diffracted beams as a function of some incidence initial condition (energy or angle). Like TRs, they take place when the scattered particle undergoes a free motion parallel to the surface before leaving it, but the perpendicular bounded one is characterized by having an energy equal to one of the bound levels of the laterally averaged potential (here assumed to be a Morse function). This temporary vibrational trapping process is ruled by the internal width of the resonance. ${ }^{9}$ Although this classical picture for a SAR has been widely discussed and accepted in the literature, long time ago we showed ${ }^{7,10}$ that this is not always the case, as clearly seen in Fig. 2, where the third resonance appears below the trapping region, in the single scattering regime. 


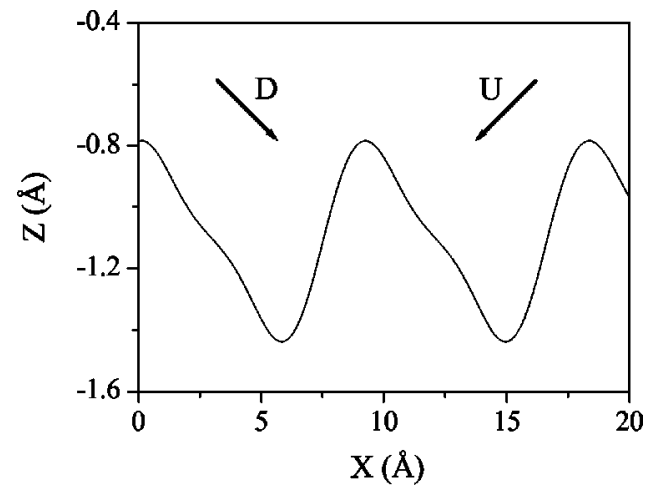

FIG. 3. Interaction potential contour at $E_{i}=21 \mathrm{meV}$ for the $\mathrm{He}-\mathrm{Cu}(117)$ system. The downhill (D) and uphill (U) incidence directions are indicated by arrows.

On the other hand, it has been shown for stepped surfaces that the corresponding dynamics is not parity and timereversal invariant, ${ }^{11}$ and diffraction and resonance patterns are strongly modified depending on the way the incoming particles approach the surface (see Fig. 3), i.e., the process strongly depends on whether downhill $\left(\theta_{i}>0\right)$ or uphill $\left(\theta_{i}\right.$ $<0)$ scattering conditions are considered. Experimentally, recent diffraction measurements on $\mathrm{He}$ scattering off the $\mathrm{Rh}(311)$ surface have been reported. ${ }^{12}$

In order to better understand TRs and SARs within a fully quantum and (at the same time) causal context, we resort to Bohmian mechanics. ${ }^{13,14}$ This theory combines both the accuracy of the standard quantum description and an intuitive insight derived from a formalism based on welldefined trajectories. Therefore, it constitutes a powerful theoretical framework to understand the physics underlying microscopic phenomena, which has been recently applied with success to relevant phenomena in surface scattering and molecular dynamics. ${ }^{7,15}$ For example, Wyatt and co-workers ${ }^{16,17}$ have analyzed the scattering of wave packets off barriers, elucidating the mechanisms leading to tunneling and resonance effects in terms of quantum trajectories. In mixed quantum-classical simulations, Prezhdo and Brooksby ${ }^{18}$ have studied the problem of quantum backreaction for the $\mathrm{O}_{2}-\mathrm{Pt}$ interaction, and Gindensperger et al. have reported a study including continuum states, ${ }^{19}$ and a description of rotational diffractive scattering. ${ }^{20}$ Moreover, efforts are also being devoted to solve directly the Bohmian equations of motion by means of approximate quantum potentials, ${ }^{21}$ applying successfully this technique to different problems in molecular dynamics.

Since the classical $\mathrm{He}-\mathrm{Cu}(117)$ system displays a chaotic or a regular dynamics depending on the incidence conditions, ${ }^{22}$ it is also expected a priori that quantum trajectories manifest reminiscent features of those dynamics. In particular, a Bohmian representation of the skipping orbits will also be provided. According to a hydrodynamical picture (and through the quantum-classical correspondence between quantum and classical trajectories ${ }^{7,15}$ ), a classical regular dynamics translates into a laminar motion regime for quantum trajectories. On the other hand, a classical chaotic dynamics corresponds to a more turbulent motion regime, which may lead to a long-term temporary trapping of quantum trajectories. However, unlike the quantum dynamics associated to the $\mathrm{He}-\mathrm{CO} / \mathrm{Pt}(111)$ system, ${ }^{23,24}$ a strong (quantum) vorticial regime is not expected. The weak corrugation of the $\mathrm{Cu}$ surface in comparison with that induced locally by a single $\mathrm{CO}$ adsorbate on the Pt surface and its periodicity, which highly diminishes the effects of the superposition between different types of outgoing wave fronts [circular and plane wave fronts in the case of the $\mathrm{He}-\mathrm{CO} / \mathrm{Pt}(111)$ system], avoid the formation of a well-defined vorticial or nodal structure with bunches of (quantum) trajectories spinning around the vortices.

The organization of this paper is as follows. In Sec. II we briefly introduce the fundamentals of Bohmian mechanics that will allow to interpret the results obtained. In Sec. III the working model is described. In Sec. IV we present and discuss our classical and quantum calculations for the scattering of He atoms off the $\mathrm{Cu}(117)$ surface. Finally, in Sec. V the main conclusions arisen from this work are summarized.

\section{NOTIONS OF BOHMIAN MECHANICS}

The fundamental equations of motion in Bohmian mechanics are derived by substituting the wave function in polar form,

$$
\Psi_{t}(\mathbf{r})=\rho_{t}^{1 / 2}(\mathbf{r}) e^{i S_{t}(\mathbf{r}) / \hbar},
$$

where $\rho_{t}$ and $S_{t}$ are two real-valued functions, into the timedependent Schrödinger equation, thus obtaining (in units of $\left.\hbar^{2} / 2 m\right)$

$$
\begin{aligned}
& \frac{\partial \rho_{t}}{\partial t}+\nabla \cdot\left(\rho_{t} \frac{\nabla S_{t}}{m}\right)=0, \\
& \frac{\partial S_{t}}{\partial t}+\frac{\left(\nabla S_{t}\right)^{2}}{2 m}+V-\frac{\nabla^{2} \rho_{t}^{1 / 2}}{\rho_{t}^{1 / 2}}=0,
\end{aligned}
$$

which are the continuity and quantum Hamilton-Jacobi equations, respectively; here $V$ is the (classical) gas-surface interaction potential and the last term in the left-hand side (lhs) of Eq. (5b) is the so-called quantum potential $Q_{t}$. This contextdependent, nonlocal potential determines together with $V$ the total force acting on the system.

The correspondence principle in this formalism is mathematically expressed as $Q_{t} \rightarrow 0$, which leads to a continuous, smooth transition from quantum to classical mechanics provided that the dependence of $S_{t}$ on $\rho_{t}$ can be neglected. ${ }^{25}$ Otherwise the evolution of $S_{t}$ is influenced by the quantum state through $Q_{t}$, and the individual motion of each particle is strongly determined by the evolution of the whole ensemble. This implies, for example, that a redefinition or generalization of the concept of asymptotic region in scattering theory is needed. According to classical criteria, it is usually defined as the spatial region where the action of (classical) external forces on particles is negligible $(V \simeq 0)$, so that they undergo a free motion. However, within Bohmian mechanics, forces acting on particles are also determined by $Q_{t}$, which in general does not approach zero far from the interaction region, as a typical classical potential does, since it depends on the curvature of $\rho_{t}$ rather than its absolute value. 
The action of $Q_{t}$ extends far beyond the classical asymptotic region, and hence the condition for free quantum motion (i.e., vanishing effective potential, $V_{\text {eff }}=V+Q_{t} \simeq 0$, in analogy to classical mechanics) only holds locally. In atom-surface scattering problems, ${ }^{26}$ for example, this condition fulfills along the directions defining the diffraction channels.

Analogously to the classical Hamilton-Jacobi theory, in Bohmian mechanics the trajectories associated to the state (4) satisfy

$$
\mathbf{p}_{t}=\nabla S_{t}=\frac{\hbar}{2 i} \frac{\Psi_{t}^{*} \nabla \Psi_{t}-\Psi_{t} \nabla \Psi_{t}^{*}}{\left|\Psi_{t}\right|^{2}},
$$

where $\mathbf{p}_{t}=m \dot{\mathbf{r}}_{t}$. From Eq. (6) it is clear that in order to compute the quantum trajectories one needs either to solve Eqs. (5) or to obtain $\Psi_{t}$ through the time-dependent Schrödinger equation. Here we have considered the second procedure, although there are works focused on the first one. ${ }^{15-17,21}$ This procedure allows a better visualization of Bohm's former idea that a quantum state is completely specified by considering both $\Psi_{0}$ and $\mathbf{r}_{0}$ at some initial time; then, the wave and the particle evolve simultaneously in a deterministic way. ${ }^{13}$ This evolution is special since $\Psi_{t}$ "guides" the particle's motion, but there is no reciprocal action of the particle on the wave (this is the so-called problem of the backreaction ${ }^{18}$ in Bohmian mechanics). On the other hand, the statistical predictions of the standard quantum mechanics are recovered in Bohmian mechanics by considering an ensemble of non-interacting particles with initial positions randomly distributed and weighted according to the initial probability density $\left(\rho_{0}=\left|\Psi_{0}\right|^{2}\right)$.

Due to the similarity between Eq. (6) and its classical counterpart, it is expected that quantum trajectories display certain features resembling the dynamics determined by $V$. This fact is better understood if we assume that $\Psi_{t}$ depends explicitly on $\hbar$. Then, it is possible to derive an expression for Eq. (6) in terms of the corresponding classical equation of motion. ${ }^{7,15}$ Thus, the wave function (4) is first written as

$$
\Psi_{t}=e^{i \bar{S}_{t} / \hbar},
$$

with

$$
\bar{S}_{t}=\sum_{n=0}^{\infty}\left(\frac{\hbar}{\mathrm{i}}\right)^{n} \bar{S}_{t}^{(n)} .
$$

The function $\bar{S}_{t}$ is related to $\rho_{t}$ and $S_{t}$ as

$$
\begin{aligned}
& \rho_{t}^{1 / 2}=\exp \left[\sum_{n=0}^{\infty}(-1)^{n} \hbar^{2 n} \bar{S}_{t}^{(2 n+1)}\right], \\
& S_{t}=\sum_{n=0}^{\infty}(-1)^{n} \hbar^{2 n} \bar{S}_{t}^{(2 n)} .
\end{aligned}
$$

Substituting Eq. (9b) in Eq. (6) one obtains

$$
\mathbf{p}_{t}=\mathbf{p}_{t}^{(\mathrm{cl})}+\sum_{n=1}^{\infty}(-1)^{n} \hbar^{2 n} \nabla \bar{S}_{t}^{(2 n)}
$$

where $\mathbf{p}_{t}^{(\mathrm{cl})}=\nabla \bar{S}_{t}^{(0)}$ is the classical law of motion since $\bar{S}_{t}^{(0)}$ is the classical action. ${ }^{7,15}$ Therefore, by considering an explicit dependence of $\Psi_{t}$ on $\hbar$, we observe that quantum trajectories can be interpreted as classical ones "dressed" with a series of terms describing the quantum interference effects [given by the second term in the right-hand side (rhs) of Eq. (10)]. These "dressing" terms constitute the capital difference between both types of trajectories, and in general (i.e., if there is no explicit dependence on $\hbar$ ) it is not possible to reduce the classical law of motion from the generalized quantum one by any smooth limiting process. ${ }^{25}$ Nonetheless, Eq. (10) clearly shows that classical mechanics underlies quantum mechanics, and hence each quantum phenomenon should display some typically classical features (provided that they are not totally suppressed by the interference terms, of course). This fact has been observed, for example, in the Bohmian generalization of classical rainbows ${ }^{26}$ and permanent trapping. ${ }^{24}$ In the following we will analyze other interesting classical "fingerprints" observed within this Bohmian description of atom-surface scattering.

\section{THE POTENTIAL MODEL}

Here we have chosen the $\mathrm{He}-\mathrm{Cu}(117)$ system as a working model to study elastic resonance effects. This scattering process is characterized by a very weak (essentially negligible) corrugation along one of the surface axes (say the $y$ axis in a reference framework where $x$ and $z$ are the axes along the parallel and perpendicular directions to the surface, respectively), so that the Hamiltonian can be written as

$$
H=\frac{1}{2 m}\left(p_{x}^{2}+p_{z}^{2}\right)+V(x, z),
$$

where $m$ is the mass of a $\mathrm{He}$ atom. The two-dimensional interaction potential (see Fig. 3) modeling the $\mathrm{He}-\mathrm{Cu}(117)$ scattering ${ }^{27,28}$ consists of the sum of a Morse function

$$
V_{M}(z)=D\left[e^{-2 \alpha z}-2 e^{-\alpha z}\right],
$$

with $D=6.35 \mathrm{meV}$ and $\alpha=1.05 \AA^{-1}$, and a coupling term

$$
\begin{aligned}
V_{C}(x, z)= & D e^{-2 \alpha z} \sum_{n=1}^{4}\left[v_{n}^{r} \cos \left(\frac{2 \pi n x}{a}\right)\right. \\
& \left.+v_{n}^{i} \sin \left(\frac{2 \pi n x}{a}\right)\right],
\end{aligned}
$$

with $a=9.12 \AA$ being the unit cell length, and $v_{n}^{r}$ and $v_{n}^{i}$ the real and imaginary parts, respectively, of the complex coefficients: $v_{1}=(0.1828,-0.0863), v_{2}=(0.0593,0.0157), v_{3}$ $=(0.0116,0.0002)$, and $v_{4}=(0.0017,0.0010)$, taken from literature. $^{3,29}$

\section{RESULTS AND DISCUSSION}

In order to simulate with good accuracy the scattering process, we have used an initial incoming plane wave (approximated by a linear superposition of 250 Gaussian wave packets ${ }^{15}$ homogeneously distributed along an axis perpendicular to the incident wave vector) covering a length of five unit cells during its collision with the surface. This plane wave is launched with an initial momentum $k_{i}=6.34 \AA^{-1}$ $\left(E_{i}=21 \mathrm{meV}\right.$ and $\left.\lambda_{\mathrm{dB}}=0.991 \AA\right)$ from the classical asymptotic region, that is, from a distance such that the wave closest border to the surface is at $12 \AA$, where $V \simeq 0$. Quan- 
tum trajectories are obtained by numerical integration of Eq. (6) once the time-dependent Schrödinger equation is solved by a standard spectral method. ${ }^{30}$

Here, the TR calculations have been carried out for the following incidence angles: $\theta_{\mathrm{TR}}^{(4)}=34.43^{\circ}$ for $\mathbf{N}=(4,0)$ and $\theta_{\mathrm{TR}}^{(2)}=51.51^{\circ}$ for $\mathbf{N}=(2,0)$. According to Fig. 1 , the former lays on the classical regular region, while the latter on the chaotic one. On the other hand, as can be seen in Fig. 2, at $E_{i}=21 \mathrm{meV}$ [and reciprocal lattice vector $\left.\mathbf{B}=(3,0)\right]$ SARs appear at incidence angles $\theta_{\mathrm{SAR}}^{(0)} \simeq 51^{\circ}, \theta_{\mathrm{SAR}}^{(1)} \simeq 46^{\circ}$, and $\theta_{\mathrm{SAR}}^{(2)} \simeq 43^{\circ}$ (now the superscripts make reference to the corresponding bound state), all of them very close around the onset of classical chaos condition, $\theta_{i} \simeq 44.75^{\circ}$. Since $\theta_{\mathrm{SAR}}^{(0)}$ $\simeq \theta_{\mathrm{TR}}^{(2)}$, SAR conditions will also be discussed when analyzing such a TR condition.

\section{A. Classical dynamics: Direct scattering and skipping orbits}

Classically, depending on the incidence conditions, the $\mathrm{He}-\mathrm{Cu}(117)$ system can show two different dynamical regimes: regular and chaotic. ${ }^{22}$ The transition from the former to the latter takes place when the rainbow angle exceeds the critical value $\pm \pi / 2$. The chaotic dynamics is characterized by a temporary vibrational trapping of $\mathrm{He}$ atoms, and the different bouncing motions along the surface are known as skipping orbits. ${ }^{6,7}$ Atoms keep moving in these orbits until the effective momentum exchange between the $x$ and $z$ directions allows them to overcome the attractive term of the interaction potential and escape. This dynamics contrasts with that found in $\mathrm{He}-\mathrm{CO} / \mathrm{Pt}(111)$ scattering. ${ }^{23,24}$ Due to the weak corrugation of the Pt surface, atoms remain indefinitely trapped along the surface unless other adsorbates favor such an effective momentum exchange, thus allowing them to escape.

The classical dynamics of the $\mathrm{He}-\mathrm{Cu}(117)$ system is well characterized by the deflection function, which relates the final deflection angle of atoms, $\theta_{f}$, to their respective impact parameters for given incidence conditions $\left(E_{i}, \theta_{i}\right)$. Since classical dynamics is local and the system possesses translational symmetry along the $x$ direction, all the dynamical information can be obtained from the behavior of the deflection function along a single unit cell (this is the reason why normalized impact parameters are used). The corresponding deflection functions for downhill scattering at the two TR conditions mentioned above are displayed in Fig. 4. In Fig. 4(a) the presence of four rainbow angles (three positive and one negative) is clearly observed as extrema of the deflection function. The smoothness of this function is the typical feature of a classical regular regime. In Fig. 4(b), with a greater value of the incidence angle, the three (positive) rainbow angles exceed $+\pi / 2$, and then a chaotic dynamics (ill behavior of the classical deflection function) leading to a temporary vibrational trapping of atoms along the surface occurs. This region, far from presenting randomly distributed deflection angles, has a fractal structure. ${ }^{7}$ For uphill scattering, a chaotic dynamics is observed in both cases. However, here it is caused because the lowest rainbow angle (now there is a downwards shift of the deflection function, and all rainbow angles are negative) exceeds $-\pi / 2$. This is a
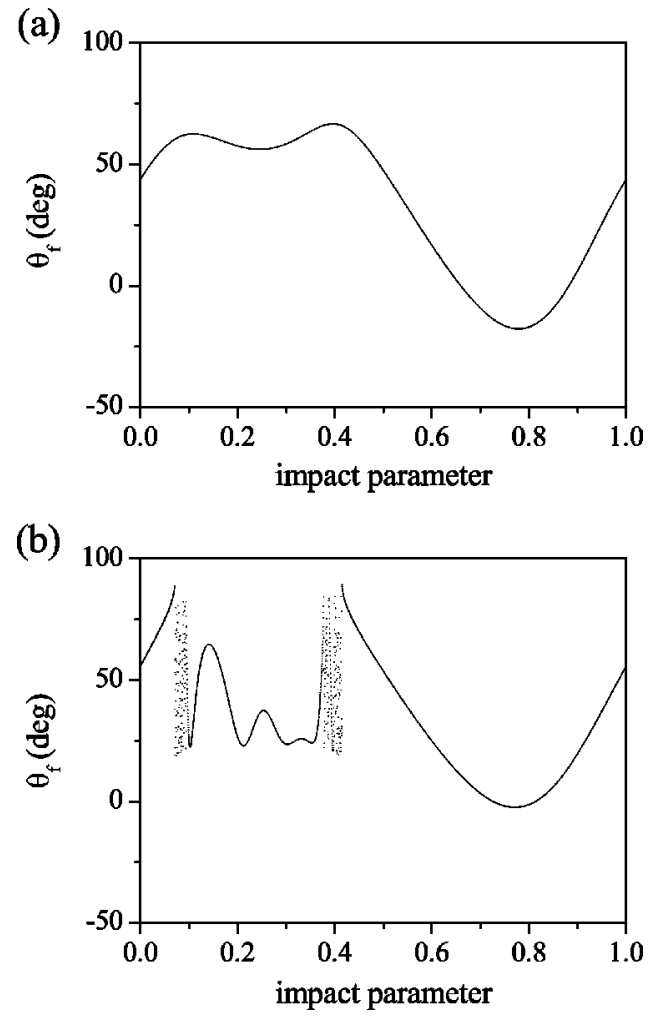

FIG. 4. Classical deflection function for $\mathrm{He}-\mathrm{Cu}(117)$ scattering at downhill incidence conditions $E_{i}=21 \mathrm{meV}$, and (a) $\theta_{i}=\theta_{\mathrm{TR}}^{(4)}$ and (b) $\theta_{i}=\theta_{\mathrm{TR}}^{(2)}$. The impact parameter is normalized to the length of one unit cell ( $a$ $=9.12 \AA$ ).

clear manifestation of the nonreversal space symmetry of the $\mathrm{Cu}(117)$ surface. The lack of symmetry implies that the effective momentum exchange from the incidence to the parallel direction is more efficient in the uphill scattering, thus inducing temporary trapping in the case of both incidence conditions.

In order to compare later both classical and Bohmian dynamics quantitatively, it is important to have some measurement providing more information about the classical trapping. This information can be obtained, for example, from the fraction of trajectories, $\mathcal{N}_{t}$, remaining inside a certain region $\Sigma$ as a function of time. The function $\mathcal{N}_{t}$ is shown in Fig. 5 for the two incidence conditions considered above and for both downhill and uphill scatterings. It has been obtained by counting the number of trajectories remaining inside the region $\Sigma:=\left\{z \leqslant 8 \AA\right.$ at time intervals of 0.5 ps. $\mathcal{N}_{t}$ has been smoothed by using $B$-splines (dashed lines). These trajectories are initially distributed along a line perpendicular to the incidence direction (no time delay effects derived from classical trajectories started either closer to or farther from the surface are considered now; see below), and in the classical asymptotic region. The plateau of value 1 reached by $\mathcal{N}_{t}$ indicates that all trajectories are inside $\Sigma$, and remains for $\approx 1.5 \mathrm{ps}$ independently of the dynamics (downhill or uphill). The time at which this plateau is reached depends, of course, on the initial angle. Trajectories launched at $\theta_{i}=\theta_{\mathrm{TR}}^{(2)}$ undergo a delay of $0.5 \mathrm{ps}$ approximately in their entry to and exit from $\Sigma$ with respect to those launched at $\theta_{i}=\theta_{\mathrm{TR}}^{(4)}$. The collision time for a trajectory to reach its first turning point on 

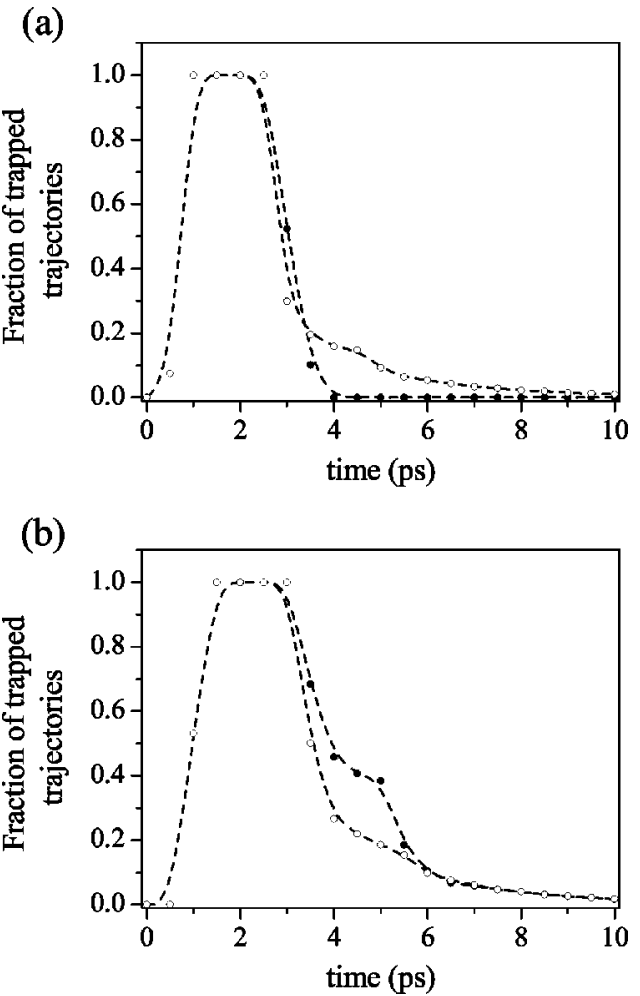

FIG. 5. Fraction of trapped classical trajectories inside the region $\Sigma$ for incidence conditions $E_{i}=21 \mathrm{meV}$, and (a) $\theta_{i}=\theta_{\mathrm{TR}}^{(4)}$ and (b) $\theta_{i}=\theta_{\mathrm{TR}}^{(2)}$. Downhill and uphill scatterings are indicated by solid and open circles, respectively. In all cases dashed lines indicate a $B$-spline fitting to better appreciate the trend of the results.

the surface increases with the incidence angle and ranges from 0.5 to $1.8 \mathrm{ps}$ approximately for the cases considered here (uphill scattering included). However, the differences between both dynamics become more obvious from $\mathcal{N}_{t}$ as trajectories start leaving $\Sigma$. For a regular dynamics, as shown in Fig. 5(a) in the case of downhill scattering (solid circles), $\mathcal{N}_{t}$ undergoes a progressive (and relatively fast) decrease until about $4 \mathrm{ps}$, when it vanishes completely because all trajectories are outside $\Sigma$. On the contrary, a chaotic dynamics, as that displayed by downhill scattering at $\theta_{i}=\theta_{\mathrm{TR}}^{(2)}$, induces temporary trapping of trajectories, thus leading to a slower decrease of $\mathcal{N}_{t}$ [see Fig. 5(b)]. Up to 4 ps approximately the decrease is relatively fast, like in the regular case. Then, for about $1 \mathrm{ps}, \mathcal{N}_{t}$ shows a slight shoulder indicating that although there are trajectories exiting at a slow rate, an important fraction of them $(\sim 40 \%)$ still remain trapped. Approximately $82 \%$ of these trapped trajectories will escape after their second collision with the surface, which corresponds to the second fast decrease in $\mathcal{N}_{t}$ observable in Fig. 5(b). After this second set of trajectories is gone, at 6 ps approximately, the decrease rate of $\mathcal{N}_{t}$ turns much slower until all He atoms get desorbed from the surface asymptotically. This change of trend in $\mathcal{N}_{t}$ with time is characteristic of a chaotic dynamics (also observable under uphill scattering conditions).

In general, for a chaotic dynamics the tail of the $\mathcal{N}_{t}$ function is expected to be smoother as the incidence angle increases, since a larger number of atoms get trapped, and therefore the escaping rate is lower. With the incidence angle, the deflection function will display wider regions of
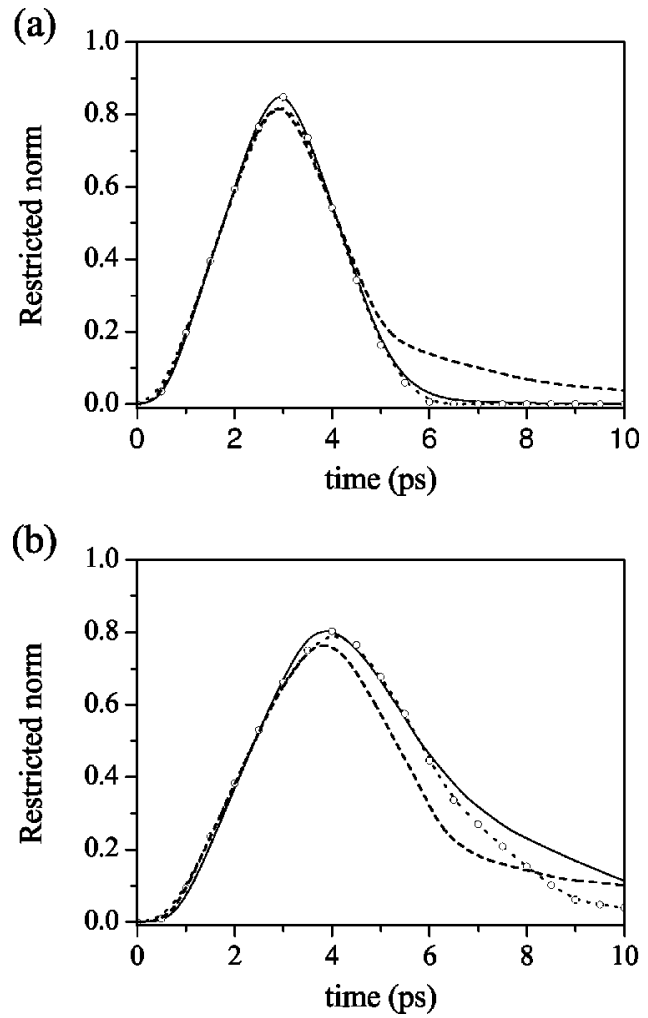

FIG. 6. Restricted norm for incoming waves with incidence conditions $E_{i}$ $=21 \mathrm{meV}$, and (a) $\theta_{i}=\theta_{\mathrm{TR}}^{(4)}$ and (b) $\theta_{i}=\theta_{\mathrm{TR}}^{(2)}$. Downhill and uphill scatterings are indicated by solid and dashed lines, respectively. For comparison, classical results are also plotted with open circles.

chaos with small windows of regularity appearing in between or at the edges of such a function.

\section{B. Wave packet calculations: The restricted norm}

The presence of different dynamical regimes and their influence on the elastic scattering can be studied from a quantum point of view by means of a very suitable analog of $\mathcal{N}_{t}$ : the restricted norm, ${ }^{27}$ defined as

$$
\mathcal{P}_{t}=\int_{\Sigma} \rho_{t} d \mathbf{r}
$$

( $\Sigma$ is the same region previously considered). The restricted norm as a function of time is displayed in Fig. 6 for incoming plane waves fulfilling TR (and nearly SAR) conditions, and downhill (solid lines) and uphill (dashed lines) scatterings. As can be seen, $\mathcal{P}_{t}$ does not reach the maximum value of 1 . This is due to the fact that there is always a fraction of the incoming wave that remains outside $\Sigma$ (smaller as the wave vector approaches the normal incidence, for which the maximum of $\mathcal{P}_{t}$ corresponds to 1 ), not contributing therefore to the integral (14). As inferred from Fig. 6, the two dynamical regimes for the $\mathrm{He}-\mathrm{Cu}(117)$ system are also evident at a quantum level. For conditions of classical regularity [Fig. 6(a) for downhill scattering] the tail of $\mathcal{P}_{t}$, after its maximum value has been reached, displays a single well-defined decrease rate. In contrast, for conditions at which classical chaotic dynamics occurs [Fig. 6(b) for downhill scattering, and both figures for uphill scattering] two different rates are 
clearly visible. The second one is smaller and corresponds, precisely, to the trapped portion of the wave packet, which moves parallel to the surface. For uphill scattering the difference between both dynamical regimes is even sharper; the two escaping rates can be better distinguished, those corresponding to the trapped portion of the wave packet being relatively lower than for downhill scattering. This fact is also observed in Fig. 5, where $\mathcal{N}_{t}$ displays similar features for uphill scattering.

For a better comparison between the classical and quantum results, the classical analog to $\mathcal{P}_{t}$ has been computed by considering a large number of classical trajectories statistically distributed according to $\rho_{0}$. These results are also plotted in Fig. 6 (open circles) for downhill scattering. As can be seen, the quantum-classical correspondence is fairly good, in particular for the regular regime [see Fig. 6(a)].

It should also be noticed that quantum trapping does not mean necessarily resonant scattering. The SAR condition rather implies a quantization of the perpendicular momentum transfer [see Eq. (3)]. In this type of calculations the transition from the single-scattering to the multiple-scattering regime is smooth and, at the onset of classical chaos, such a distinction is not very clear. The second rate has been used in Ref. 27 to compute SAR lifetimes. A more detailed analysis of these results will be given in the following section.

\section{Quantum trajectory description}

Here we analyze the structure of quantum trajectories in the two regimes represented by the incidence angles $\theta_{\mathrm{TR}}^{(4)}$ and $\theta_{\mathrm{TR}}^{(2)}$ and for both downhill and uphill scatterings. The criterion used to study the trajectories is the same followed in our previous works, ${ }^{24}$ i.e., we consider initial conditions for the trajectories distributed along cuts or slices of $\rho_{0}$ transversal to the incident wave vector direction.

In Fig. 7 a sample of quantum trajectories for the two downhill incidence conditions mentioned above, and started at slices of $\rho_{0}$ close to the surface, are displayed. In panel (a) we can observe that quantum trajectories escape through several exit channels after remaining trapped along the surface a distance of one unit cell or less. These exit channels are related, as shown in Ref. 26, to the classical caustics. On the contrary, in panel (b) quantum trajectories remain trapped along the surface for a much longer time, thus covering a larger number of unit cells. These trapped quantum trajectories represent the direct analog of the (classical) skipping orbits. The striking difference in the behavior of both types of trajectories relies on the presence of a sort of sliding motion in the region of stronger interaction in the quantum case, thus following the potential contour, while classically only a "bouncing" motion is observed for the same dynamical regime. Only a small portion of such quantum trajectories (those exiting parallel to the surface) contribute to the diffraction intensity of the corresponding TR.

We would like to stress here two more points concerning the behavior of the quantum trajectories under resonance conditions [see Figs. 2 and 7(b)]. First, a SAR in the chaotic region should be interpreted as a bounded motion along the $z$ direction with a vibrational frequency given by the corre- (a)

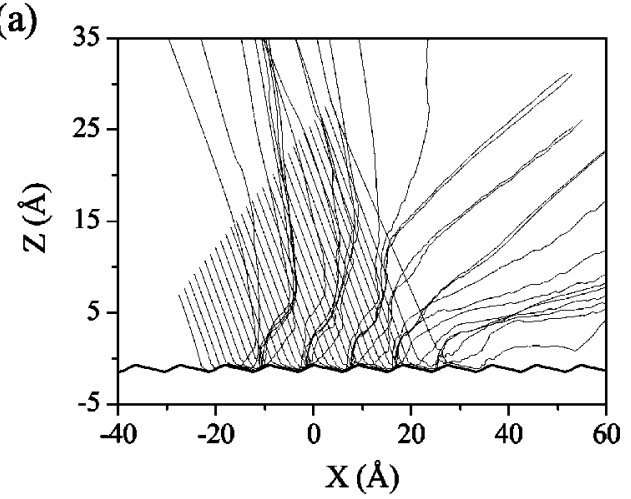

(b)

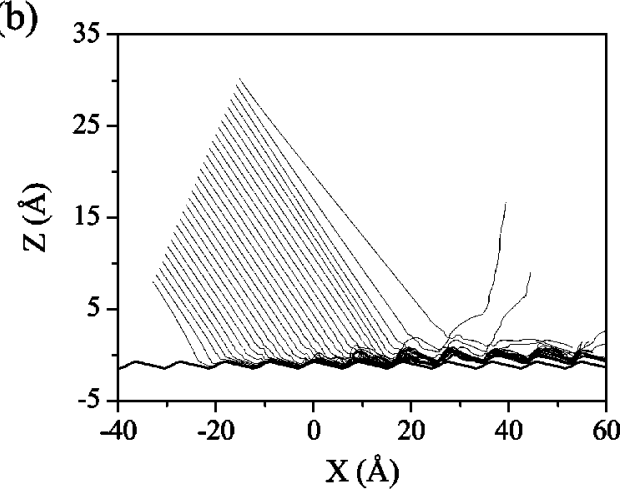

FIG. 7. Quantum trajectories for downhill scattering at incidence conditions $E_{i}=21 \mathrm{meV}$, and (a) $\theta_{i}=\theta_{\mathrm{TR}}^{(4)}$ and (b) $\theta_{i}=\theta_{\mathrm{TR}}^{(2)}$. The initial positions for the quantum trajectories are chosen along a slice in the front part of $\rho_{0}$.

sponding bound state of the interaction potential, and a free motion parallel to the surface during a lifetime given by the inverse of the internal half-width covering a distance of two or more unit cells. Second, a SAR in the regular or single scattering regime should be interpreted in a similar way, but in a shorter time scale and covering a length of one single unit cell or less. This is in sharp contrast to the classical idea of trapping, in which at least two consecutive unit cells are involved. Moreover, notice also the apparent lack of vortitial dynamics, unlike the one observed in the $\mathrm{He}-\mathrm{CO} / \mathrm{Pt}(111)$ system. ${ }^{23,24}$ Although quantum trapping is observed in the lower panel, due to the weak corrugation of the $\mathrm{Cu}$ surface and its periodicity a well-defined structure of quantum vortices cannot be distinguished. Nonetheless, certain degree of vorticity can be still appreciated very close to the surface.

In Fig. 8 quantum trajectories for the two uphill scattering conditions are also plotted, showing again the non parity and time-reversal invariance of this scattering. In both cases the existence of a new type of quantum skipping orbits is apparent. Particles usually remain moving along the surface. However, as can be seen, some of them are now bouncing along a different axis far from the surface. It could be said that they are feeling an effective corrugated (quantum) potential along those axes. This effect is more clearly observed in Fig. 8(a) at final grazing angles.

In order to better understand the concept of trapping in the classical and quantum domains, nine quantum and classical trajectories sampling the region covered by the incoming plane wave will be analyzed in more detail. Accordingly, we have chosen three initial conditions in the rear, central, 
(a)

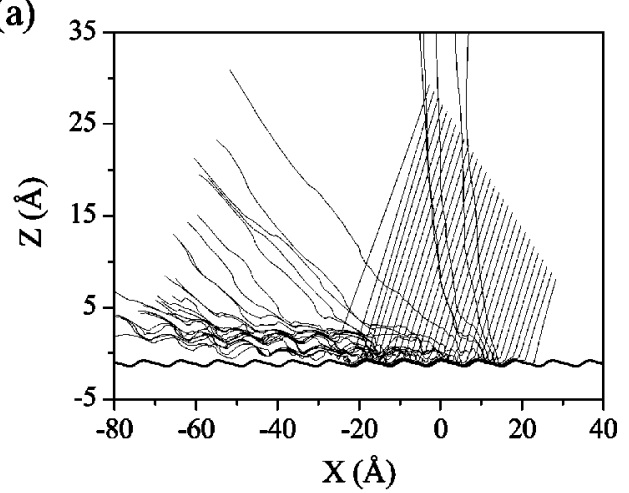

(b)

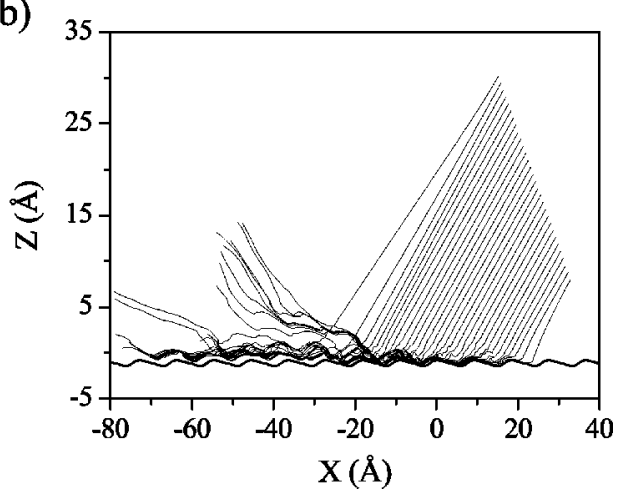

FIG. 8. Quantum trajectories for uphill scattering at incidence conditions $E_{i}=21 \mathrm{meV}$, and (a) $\theta_{i}=-\theta_{\mathrm{TR}}^{(4)}$ and (b) $\theta_{i}=-\theta_{\mathrm{TR}}^{(2)}$. The initial positions for the quantum trajectories are chosen along a slice in the front part of $\rho_{0}$.

and front parts of $\rho_{0}$, and incidence at $\theta_{i}=\theta_{\mathrm{TR}}^{(2)}$. In Fig. 9 the behavior of such nine quantum trajectories (left column) is compared to those of their classical counterparts (right column). A first remarkable feature observed is the global (or nonlocal) character of Bohmian dynamics in contrast to the local character of classical dynamics. The different quantum behavior of atoms as they approach the surface with different initial conditions is caused by the quantum potential. Observe that classical dynamics is independent of the initial distribution of the particles for the same initial positions. The quantum dynamics is very strongly influenced by the initial distribution: particles coming from behind "know" (through the information transmitted by the quantum potential) that before them there are other particles reaching the surface, and therefore they cannot follow the same tracks. In this way, while the front trajectories reach the surface and move along it [see Fig. 9(c)], those started behind cannot approach it [see Figs. 9(a) and 9(b)]. Indeed, the trajectories started in the outmost rear part of $\rho_{0}$ can approach (in average) only at 6.4 $\AA$, which is a remarkable distance taking into account that the upper boundary of $\Sigma$ is $8 \AA$. This behavior making the atoms to undergo a bounce when they are still far from the surface, arises from the different effective forces that they "feel" depending on their initial position relative to $\rho_{0}$. The concept of quantum pressure was introduced in another context $\mathrm{t}^{24}$ in order to explain this observation. Particles under a high pressure (and close to the surface) are constrained to keep moving along the surface until such a pressure decreases enough to let them escape. Thus, as can be inferred
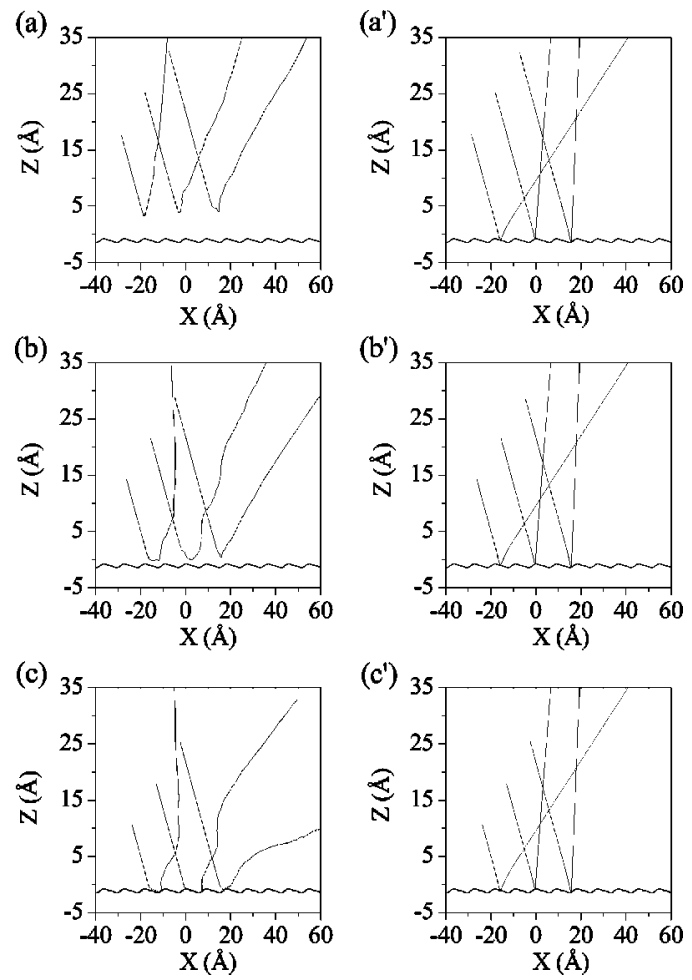

FIG. 9. Quantum (left) and classical (right) trajectories started with initial positions covering the rear (a), central (b), and front (c) parts of $\rho_{0}$ for downhill $\mathrm{He}-\mathrm{Cu}(117)$ scattering at $\theta_{\mathrm{TR}}^{(2)}$ and $E_{i}=21 \mathrm{meV}$.

from Fig. 7(a), the effects of quantum pressure are relatively small along the exit channels. On the other hand, quantum trapping also comes from the attractive dynamics governed by the interaction potential (which can coincide with conditions of classical trapping). In Fig. 7(b) it is apparent that the front trajectories follow a sliding motion along the potential surface in the region where the collision of the whole ensemble of trajectories with the surface takes place. However, when the upper trajectories begin to leave, such a pressure decreases, and the quantum trajectories that remain trapped along the surface either keep moving in the same direction due to the attractive potential or simply escape. Second, let us emphasize in the regular and chaotic regimes the impulsive character of classical trajectories. In classical mechanics the collision can be considered, in a simplistic way, as an instantaneous kick of the particle against the surface, thus changing its initial momentum. Depending on this initial momentum and how the kick takes place (i.e., depending on the particular orientation of the surface with respect to the direction of the incoming particle), the atom will get trapped (and move along a length of more than one unit cell) or not. This is something totally different with respect to what happens in Bohmian mechanics, where the concepts of collision and trapping acquire a more generalized meaning. Only those trajectories associated to the central and rear parts of $\rho_{0}$ will display features typical of classical trajectories. And third, in the light of the previous statements, we note that for $\mathrm{He}$ atoms scattering off $\mathrm{Cu}(117)$, in particular, and any corrugated surface, in general, the quantum dynamics can be considered as an isomorphism of the classical one "plus interferences." Thus, classical dynamics provides a sort of pattern 
ruling the different observable dynamics (regular or chaotic, in this case), and interferences determine the final motion displayed by the quantum trajectories, i.e., their global dynamics under the effects of the quantum pressure.

Finally, the quantum results shown in Fig. 6 can also be obtained by using an ensemble of Bohmian trajectories initially distributed according to $\rho_{0}$ (as in the classical case, also shown in that figure). Due to Eq. (5a) and its coupling to Eq. (5b), the distribution of Bohmian trajectories at any time $\rho_{t}$ will be equal to $\left|\Psi_{t}\right|^{2}$. Therefore, $\mathcal{P}_{t}$ must be exactly the same independently of how it was obtained, either by Bohmian or by standard quantum mechanics. No difference (apart from those derived from the accuracy of the different computational techniques, obviously) can be observed between both calculations; otherwise, any physical implication derived from such a difference will be undoubtedly wrong. Thus, by computing the lifetimes (either by Bohmian or standard quantum calculations) as suggested in Ref. 27, i.e., fitting the tail of the restricted norm to an exponentially decrease function, we have obtained $\tau \simeq 2.44 \mathrm{ps}$ for $\theta_{i}=\theta_{\mathrm{TR}}^{(2)}$ (SAR condition). From Fig. 2, an estimation of 2.13 ps is obtained for the same resonance. On the other hand, for off resonance conditions, for example, at $\theta_{i}=\theta_{\mathrm{TR}}^{(4)}$, the residence time is about $0.57 \mathrm{ps}$.

\section{Quantum deflection functions}

In Figs. 10 and 11 the quantum deflection functions for downhill scattering at the two incidence conditions used above are displayed. In each plot, a total number of 5000 trajectories with initial positions distributed along the same three different transversal slices of $\rho_{0}$ as in Fig. 9 have been used. The impact parameter is given in terms of the total length illuminated by the atomic beam (five unit cells). Let us remark that the quantum deflection function has been evaluated in the Fresnel region, where the classical asymptotic region is already reached, in order to avoid longer propagation times. Hence, the typical staircase structure presented by this function in the Fraunhofer region, 25,26 where each step corresponds to a different diffraction channel, is not observable. Moreover, due to the off-normal incidence and the lack of reflection symmetry of the interaction potential, one should not expect that the trajectories associated to each slice of $\rho_{0}$ give rise to similar quantum deflection functions, as happens in simpler cases. ${ }^{26}$ Thus, the step corresponding to a certain diffraction channel will show a different length depending on the deflection function considered due to the non (dynamical) equivalence among trajectories started at different slices.

In general, three features are remarkable from the quantum deflection function. First, the global nature of the quantum dynamics is again patent. From top to bottom, we can observe in each figure that the corresponding dynamics has its own peculiarities. In the upper panels, which represent the dynamics of the trajectories initiated farther from the surface, the deflection function is relatively smooth, like in a laminar regime. This is an indication of a low quantum pressure. On the contrary, as we approach the surface and the quantum pressure increases, such a smooth behavior turns into a more
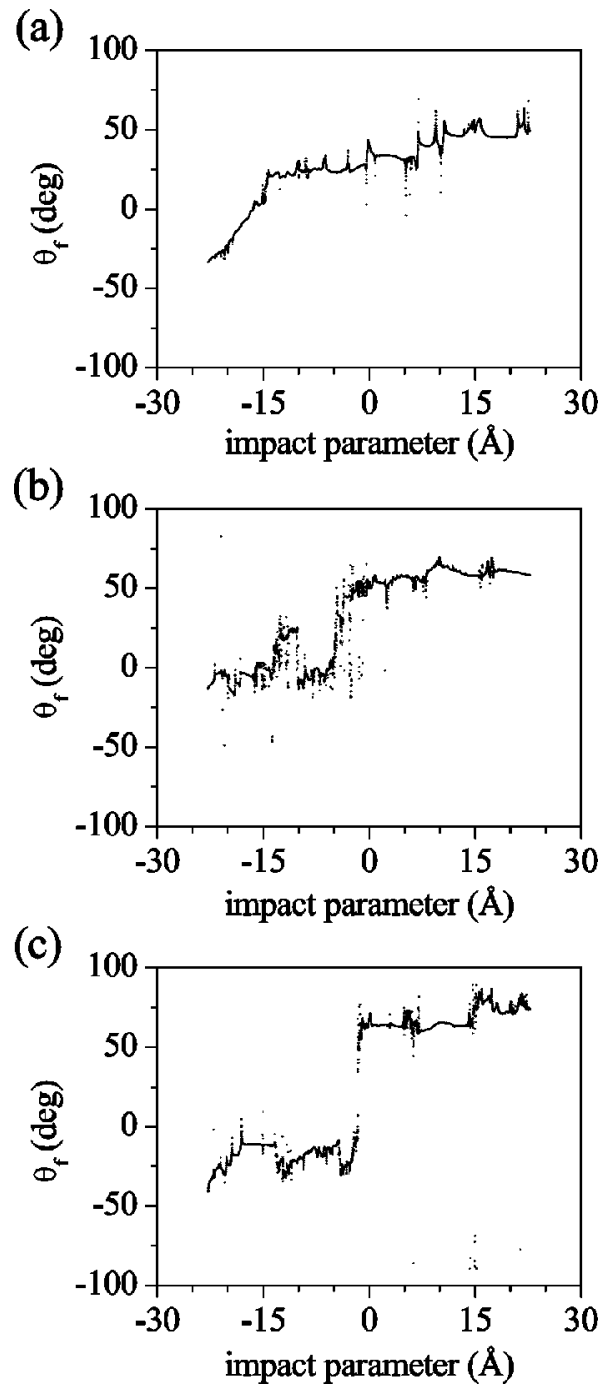

FIG. 10. Quantum deflection function computed with quantum trajectories started with initial positions along the rear (a), central (b), and front (c) parts of $\rho_{0}$ for downhill $\mathrm{He}-\mathrm{Cu}(117)$ scattering at $\theta_{\mathrm{TR}}^{(4)}$ and $E_{i}=21 \mathrm{meV}$.

complex one. Second, also from top to bottom, it is apparent which groups of trajectories are subsequently going to mainly contribute to each diffraction peak. This is clearer in the case of Fig. 10. The trajectories initiated farther from the surface [see Fig. 10(a)] will mainly contribute to the peaks with higher final scattering angles; the $+\pi / 2$ value is not reached. Trajectories starting at the central part of $\rho_{0}$ [see Fig. 10(b)] will cover more or less the same final angular region as before. Finally, trajectories in the closest part to the surface [see Fig. 10(c)] will contribute to two totally different regions: negative final scattering angles and the $(4,0)$ TR (quantum skipping trajectories). Therefore a mapping can be established between the different regions covered by the initial plane wave and those of the diffraction and/or resonance patterns. Third, from Fig. 4(a), it is seen that for the same initial conditions, classical trajectories do not display chaos, and this leads to a certain smoothness in the deflection functions calculated, as shown in Fig. 10. However, the structure of the quantum deflection functions plotted in Fig. 11 for $\theta_{i}=\theta_{\mathrm{TR}}^{(2)}$ at $E_{i}=21 \mathrm{meV}$ is much more involved. In particular, in the lowest panel, we observe that the trajectories ini- 
(a)

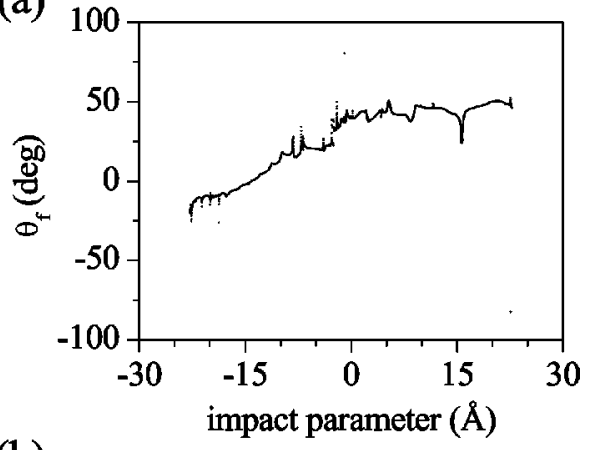

(b)

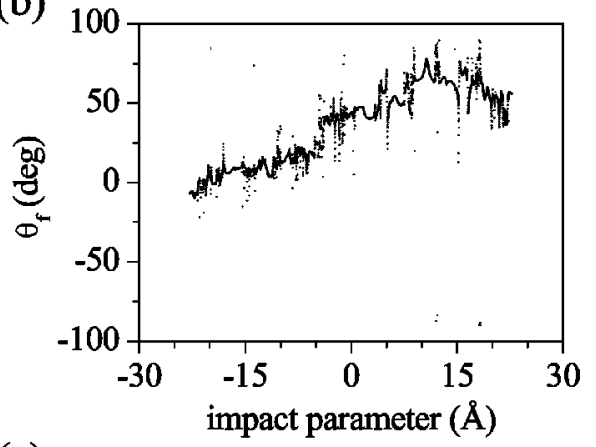

(c)

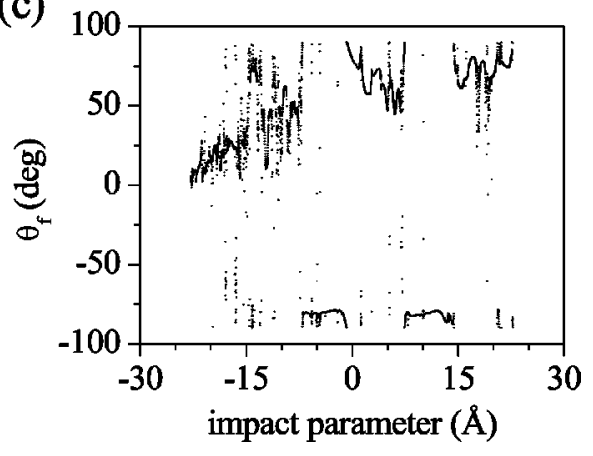

FIG. 11. Quantum deflection function computed with quantum trajectories started with initial positions along the rear (a), central (b), and front (c) parts of $\rho_{0}$ for downhill $\mathrm{He}-\mathrm{Cu}(117)$ scattering at $\theta_{\mathrm{TR}}^{(2)}$ and $E_{i}=21 \mathrm{meV}$.

tiated closer to the surface cover mainly the whole final angular range (from $-\pi / 2$ to $\pi / 2$ ). This high complexity displayed by the quantum deflection function is a clear manifestation of the underlying classical chaotic scattering dynamics [see Fig. 4(b)] caused by the temporary trapping of trajectories along the surface. Nonetheless, in the Bohmian case it might also be expected that for a longer propagation time interferences will play an important role with respect to the final asymptotic shape of the quantum deflection function. Limitations in the size of the interferences due to the uncertainty principle a priori could restrict the fractal behavior of the quantum dynamics, in opposition to what can be observed classically. In some sense, this is similar to what happens in a double-slit experiment ${ }^{7}$ when the width of the slits is much smaller than the particle incident wavelength; no portion of the incoming plane wave can pass through the slits, and the whole front is backscattered. Consequently, unlike their classical counterparts, all quantum trajectories are deflected backwards and no one passes through the slits.

In analogy to the classical trapping ratio, the fraction of trapped Bohmian trajectories $\mathcal{N}_{t}^{B}$ can also be calculated in a
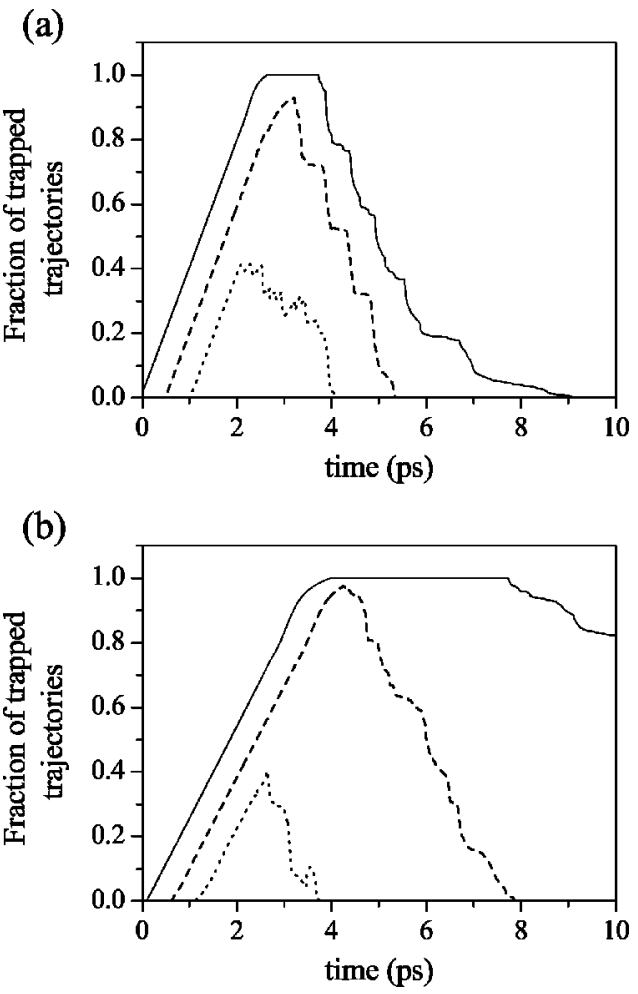

FIG. 12. Fraction of trapped Bohmian trajectories inside $\Sigma$ for downhill incidence conditions $E_{i}=21 \mathrm{meV}$, and (a) $\theta_{i}=\theta_{\mathrm{TR}}^{(4)}$ and (b) $\theta_{i}=\theta_{\mathrm{TR}}^{(2)}$. The trajectories are started with initial positions covering the rear (dotted line), central (dashed line), and front (solid line) parts of $\rho_{0}$.

similar way. This function is plotted in Fig. 12 for the three same sets of initial positions and downhill scattering used above to compute the quantum deflection functions. The contributions of the trajectories have not been weighted with the initial distribution since it is interesting to establish a one-toone comparison with $\mathcal{N}_{t}$. For this goal, we consider normalization to the highest value of $\mathcal{N}_{t}^{B}\left(\mathcal{N}_{t}^{B}=1\right.$ when all trajectories are inside $\Sigma$ ). The quantum pressure effect is again apparent. Observe that those trajectories started farther from the surface will display lower maxima for $\mathcal{N}_{t}^{B}$ than those other starting closer. Moreover, the maximum of $\mathcal{N}_{t}^{B}$ as well as the time before it gets vanished are directly linked to the initial positions of the ensembles of trajectories, unlike the classical case. On the other hand, those trajectories in the front part of $\rho_{0}$ show again clear fingerprints of the classical dynamics except in the way $\mathcal{N}_{t}^{B}$ goes to 0 , which is slower; quantum pressure does not allow them to leave $\Sigma$ before other trajectories coming behind have already exited. Nonetheless, in both cases a descending staircase structure is observable [better appreciated in Fig. 12(a)]. This structure, related to the periodicity of the interaction potential seen by the incoming plane wave (one step by each unit cell illuminated), is a manifestation of the exit channels mentioned above, along which the effects of quantum pressure are smaller. As soon as a bunch of trajectories reaches one of these exit channels, $\mathcal{N}_{t}^{B}$ undergoes a sudden decrease. Thus, the main differences arise from the residence time, i.e., the time for which the maximum value of $\mathcal{N}_{t}^{B}$ is maintained. Approximately, this time is $1.09 \mathrm{ps}$ for $\theta_{i}=\theta_{\mathrm{TR}}^{(4)}$, and $3.77 \mathrm{ps}$ 

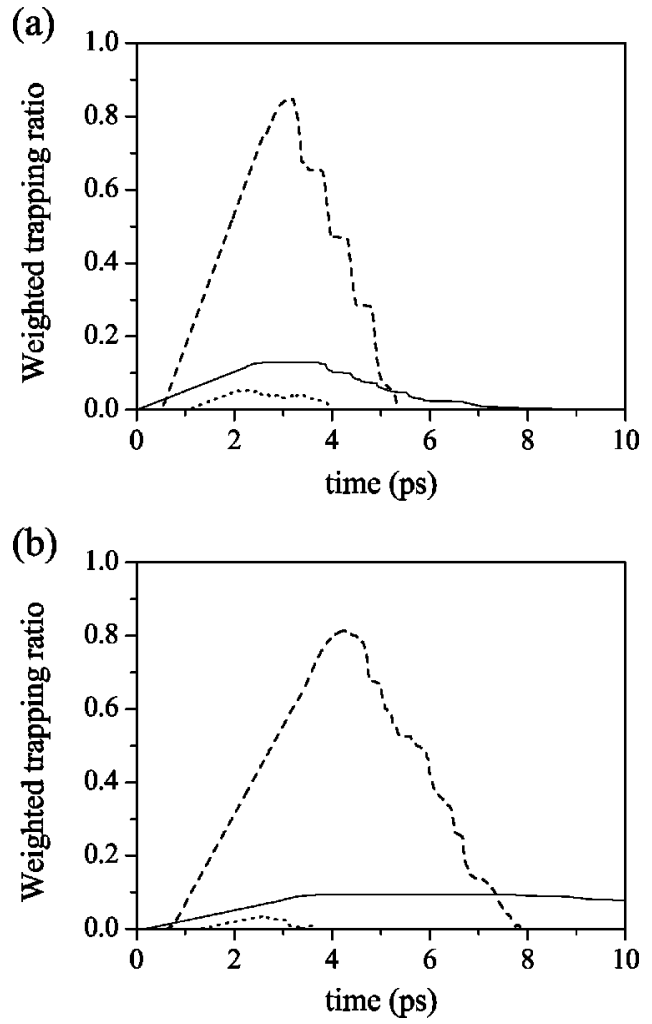

FIG. 13. Weighted fraction of trapped Bohmian trajectories inside $\Sigma$ for incidence conditions $E_{i}=21 \mathrm{meV}$, and (a) $\theta_{i}=\theta_{\mathrm{TR}}^{(4)}$ and (b) $\theta_{i}=\theta_{\mathrm{TR}}^{(2)}$, for downhill scattering. The trajectories are started with initial positions covering the rear (dotted line), central (dashed line), and front (solid line) parts of $\rho_{0}$.

for $\theta_{i}=\theta_{\mathrm{TR}}^{(2)}$. For uphill scattering conditions the residence times are longer, 1.19 and 4.10 ps, respectively.

Finally, in order to complete our analysis, the results of Fig. 12 are shown in Fig. 13 correctly weighted, so that the (statistical) relevance of the contribution of each group of trajectories to $\mathcal{P}_{t}$ and the effects of the global quantum dynamics can be better understood. In this way, we define the weighted fraction of trapped quantum trajectories as

$$
\mathcal{W}_{t} \propto \sum_{i} \rho_{0}\left(\mathbf{r}_{i}^{0}\right) \delta\left(z-z_{i}^{t}\right) \quad \text { for } z \in \Sigma,
$$

where the index $i$ runs over the total number of trajectories of each ensemble (5000), $\mathbf{r}_{i}^{0}$ is the initial position of each trajectory, and $z_{i}^{t}$ is the $z$ coordinate of each trajectory at time $t$. The proportional relation comes from the fact that the $\mathcal{W}_{t}$ function plotted is not exactly the value given by the rhs of Eq. (15), but rather a renormalized one. This renormalization is carried out by assuming the maximum value of $\mathcal{W}_{t}$ equal to the maximum value of $\mathcal{P}_{t}$. Moreover, let us remark that $\mathcal{P}_{t} \simeq \Sigma_{n} \mathcal{W}_{t}^{(n)}$, where $n$ runs over all the slices of $\rho_{0}$ considered. Thus, while $\mathcal{W}_{t}$ only accounts for the weighted number of trapped trajectories corresponding to one single slice of $\rho_{0}, \mathcal{P}_{t}$ stands for the (also weighted) total number of trapped trajectories with initial positions covering the whole spatial extension of $\rho_{0}$. Hence, in the (a) and (b) panels of Fig. 13, it is observed that each $\mathcal{W}_{t}$ function contributes differently to $\mathcal{P}_{t}$, although its shape does not change with respect to that presented by its homologous $\mathcal{N}_{t}^{B}$. As can be clearly seen, the main contribution to $\mathcal{P}_{t}$ is given by the trajectories started in the central and front slices of the region covered by $\rho_{0}$, while the rest contribute marginally. This is obvious, since the profile of the incoming plane wave along the direction parallel to the initial wave vector is a Gaussian, and then the number of trajectories in the central part of $\rho_{0}$ will be proportionally larger than in any other. Comparing Fig. 13 with the corresponding case in Fig. 6, the position of the maximum (in $t$ ) of $\mathcal{W}_{t}$ agrees fairly well with that of $\mathcal{P}_{t}$. Other ensembles of trajectories initially located at the borders of the Gaussian profile will contribute only to small deviations of the position of the maximum of $\mathcal{W}_{t}$, thus resulting a total agreement with $\mathcal{P}_{t}$. Notice, however, that the trajectories started at the front part of $\rho_{0}$ determine the long-range behavior of $\mathcal{W}_{t}$, and therefore are responsible for the tail displayed by the decaying of $\mathcal{P}_{t}$ leading to higher residence times or SAR lifetimes.

\section{CONCLUSIONS}

To summarize, in this work we have provided a full description of classical and quantum trappings in the scattering of He atoms from highly corrugated surfaces. In particular, we have studied the classical and quantum dynamics associated to the $\mathrm{He}-\mathrm{Cu}(117)$ scattering. A very well-defined quantum-classical correspondence has been established, thus generalizing the systems previously studied by the authors. $^{23,24}$ Moreover, a purely quantum description in terms of trajectories has been provided for two different kinds of resonances: threshold and selective adsorption resonances. In order to better understand such phenomena, the image of a sliding motion following the potential contour and the concept of quantum pressure have been invoked in sharp contrast to the impulsive character of classical collisions. Nonetheless, although both types of resonances are satisfactorily explained in terms of the temporary trapping of quantum trajectories, there are important differences (apart from their intrinsic physical meanings) between them related to their time and space (i.e., traveling distance along the surface) scales and to the final diffracted directions ( $\pm \pi / 2$-directions for TRs and any final direction for SARs). One of the most striking features of quantum trapping, compared to the classical one, is that it can occur within a distance of one single unit cell. Moreover, we have seen that the dynamics of sets of trajectories whose initial conditions map the different regions of the (initial) incoming wave, which are manifested in the quantum deflection function and the fraction of trapped trajectories, reveal the details about the formation of the diffraction and resonance peaks. This mapping, which cannot be provided by the standard quantum mechanics, should help to understand better the evolution of the system and its relationship with its classical analog.

\section{ACKNOWLEDGMENTS}

One of the authors (A.S.S.) wishes to acknowledge Professor Paul Brumer for support during the preparation of this manuscript. This work was supported in part by MCyT (Spain) by means of the project under Grant No. BFM20012179. 
${ }^{1}$ K. B. Whaley, C. Yu, C. S. Hogg, J. C. Light, and S. J. Sibener, J. Chem. Phys. 83, 4235 (1985).

${ }^{2}$ J. P. Toennies, J. Vac. Sci. Technol. A 2, 1055 (1984).

${ }^{3}$ D. Gorse, B. Salanon, F. Fabre, A. Kara, J. Perreau, G. Armand, and J. Lapujoulade, Surf. Sci. 147, 611 (1984); S. Miret-Artés, J. P. Toennies, and G. Witte, Phys. Rev. B 54, 5881 (1996).

${ }^{4}$ S. Miret-Artés, Surf. Sci. Lett. 366, L735 (1996); Phys. Rev. B 60, 1547 (1999).

${ }^{5}$ G. Armand and J. R. Manson, Surf. Sci. 169, 216 (1986).

${ }^{6}$ S. Miret-Artés, J. Margalef-Roig, R. Guantes, F. Borondo, and C. Jaffé, Phys. Rev. B 54, 10397 (1996); R. Guantes, F. Borondo, J. Margalef-Roig, S. Miret-Artés, and J. R. Manson, Surf. Sci. Lett. 375, L379 (1997).

${ }^{7}$ R. Guantes, A. S. Sanz, J. Margalef-Roig, and S. Miret-Artés, Surf. Sci. Rep. 53, 199 (2004)

${ }^{8}$ R. Guantes, S. Miret-Artés, and F. Borondo, Phys. Rev. B 63, 235401 (2001).

${ }^{9}$ M. Hernández, S. Miret-Artés, P. Villarreal, and G. Delgado-Barrio, Surf. Sci. 274, 21 (1992).

${ }^{10}$ F. Borondo, C. Jaffé, and S. Miret-Artés, Surf. Sci. 317, 211 (1994).

${ }^{11}$ M. Hernández, S. Miret-Artés, P. Villarreal, and G. Delgado-Barrio, Surf. Sci. Lett. 290, L693 (1993); S. Miret-Artés, Surf. Sci. 294, 141 (1993).

${ }^{12}$ D. Farías, M. Pattig, K.-H. Rieder, and J. R. Manson, Surf. Sci. Lett. 480, L395 (2001).

${ }^{13}$ D. Bohm, Phys. Rev. 85, 166 (1952).

${ }^{14} \mathrm{P}$. R. Holland, The Quantum Theory of Motion (Cambridge University Press, Cambridge, 1993).

${ }^{15}$ A. S. Sanz, F. Borondo, and S. Miret-Artés, J. Phys.: Condens. Matter 14, 6109 (2002).
${ }^{16}$ C. L. Lopreore and R. E. Wyatt, Phys. Rev. Lett. 82, 5190 (1999).

${ }^{17}$ K. Na and R. E. Wyatt, Int. J. Quantum Chem. 81, 206 (2001).

${ }^{18}$ O. V. Prezhdo and C. Brooksby, Phys. Rev. Lett. 86, 3215 (2001).

${ }^{19}$ E. Gindensperger, C. Meier, and J. A. Beswick, J. Chem. Phys. 116, 8 (2002).

${ }^{20}$ E. Gindensperger, C. Meier, J. A. Beswick, and M.-C. Heitz, J. Chem. Phys. 116, 10051 (2002).

${ }^{21}$ S. Garashchuk and V. A. Rassolov, Chem. Phys. Lett. 376, 358 (2003); J. Chem. Phys. 120, 1181 (2004); V. A. Rassolov and S. Garashchuk, ibid. 120, 6815 (2004).

${ }^{22}$ R. Guantes, F. Borondo, C. Jaffé, and S. Miret-Artés, Int. J. Quantum Chem. 52, 515 (1994); R. Guantes, F. Borondo, and S. Miret-Artés, Phys. Rev. E 56, 378 (1997).

${ }^{23}$ A. S. Sanz, F. Borondo, and S. Miret-Artés, Phys. Rev. B 69, 115413 (2004).

${ }^{24}$ A. S. Sanz, F. Borondo, and S. Miret-Artés, J. Chem. Phys. 120, 8794 (2004).

${ }^{25}$ A. S. Sanz, F. Borondo, and S. Miret-Artés, Europhys. Lett. 55, 303 (2001).

${ }^{26}$ A. S. Sanz, F. Borondo, and S. Miret-Artés, Phys. Rev. B 61, 7743 (2000).

${ }^{27}$ M. I. Hernández, J. Campos-Martínez, S. Miret-Artés, and R. D. Coalson, Phys. Rev. B 49, 8300 (1994).

${ }^{28}$ R. Guantes, S. Miret-Artés, and F. Borondo, Phys. Rev. B 63, 235401 (2001).

${ }^{29}$ D. Farías and K. H. Rieder, Rep. Prog. Phys. 61, 1575 (1998).

${ }^{30}$ R. Kosloff, in Dynamics of Molecules and Chemical Reactions, edited by R. E. Wyatt and J. Z. Zhang (Marcel Dekker, New York, 1996), pp. 185230 . 